\title{
Asymmetric Jump Beta Estimation with Implications for Portfolio Risk Management ${ }^{\text {th }}$
}

This version: 2018-04-18

\begin{abstract}
Vitali Alexeev $^{\mathrm{a}, *}$, Giovanni Urga ${ }^{\mathrm{b}, \mathrm{c}}$, Wenying Yao ${ }^{\mathrm{d}}$
${ }^{a}$ Finance Discipline Group, UTS Business School, University of Technology Sydney, Sydney NSW 2007, Australia ${ }^{b}$ Centre for Econometric Analysis, Faculty of Finance, Cass Business School, 106 Bunhill Row, EC1Y 8TZ, London, UK

${ }^{c}$ Department of Management, Economics and Quantitative Methods, University of Bergamo, Bergamo, 24127, Italy

${ }^{d}$ Department of Economics, Faculty of Business and Law, Deakin University, Burwood VIC 3125, Australia
\end{abstract}

\begin{abstract}
We evaluate the impact of extreme market shifts on equity portfolios and study the difference in negative and positive reactions to market jumps with implications for portfolio risk management. Employing high-frequency data for the constituents of the S\&P500 index over the period 2 January 2003 to 30 December 2011, we investigate to what extent the portfolio exposure to the downside and upside jumps can be mitigated. We contrast the risk exposure of individual stocks with those of the portfolios as the number of holdings increases. Varying the jump identification threshold, we show that the number of holdings required to stabilise portfolios' sensitivities to negative jumps is higher than when positive jumps are considered and that the asymmetry is more prominent for more extreme events. Ignoring this asymmetry results in under-diversification of portfolios and increases exposure to sudden extreme negative market shifts.
\end{abstract}

Keywords: Asymmetric jumps, Portfolio diversification, Systematic risk, Equity JEL Classification: C58, G11, C61

\footnotetext{
${ }^{\star 2}$ We thank Simona Boffelli, Mardi Dungey, Bart Frijns, Artem Prokhorov, George Tauchen, Viktor Todorov, Marcel Scharth, Ayesha Scott, Lars Winkelmann, Marcin Zamojski, and participants at the 10th Annual SoFiE Conference (New York, June 2017), the Midwest Finance Association Annual Meeting (Chicago, March 2017), Discipline of Business Analytics seminar at the University of Sydney (Sydney, October 2016), the Financial Econometrics and Empirical Asset Pricing Conference (Lancaster, July 2016), the Third Annual Conferences of the International Association for Applied Econometrics (Milan, June 2016), and the 2016 Auckland Finance Meeting for their valuable comments. This research is partially supported by Australian Research Council Grant DP130100168. Financial support from the Tasmanian School of Business and Economics is gratefully acknowledged. Vitali Alexeev also thanks the Centre for Econometric Analysis at the Cass Business School for hospitality and financial support during his visit in September 2015 - July 2016.

${ }^{*}$ Corresponding author

Email addresses: Vitali.Alexeev@uts.edu.au, Phone: +61 295147781 (Vitali Alexeev), G.Urga@city.ac.uk (Giovanni Urga), Wenying. Yao@deakin.edu.au (Wenying Yao)
} 


\title{
Asymmetric Jump Beta Estimation with Implications for Portfolio Risk Management
}

\begin{abstract}
We evaluate the impact of extreme market shifts on equity portfolios and study the difference in negative and positive reactions to market jumps with implications for portfolio risk management. Employing high-frequency data for the constituents of the S\&P500 index over the period 2 January 2003 to 30 December 2011, we investigate to what extent the portfolio exposure to the downside and upside jumps can be mitigated. We contrast the risk exposure of individual stocks with those of the portfolios as the number of holdings increases. Varying the jump identification threshold, we show that the number of holdings required to stabilise portfolios' sensitivities to negative jumps is higher than when positive jumps are considered and that the asymmetry is more prominent for more extreme events. Ignoring this asymmetry results in under-diversification of portfolios and increases exposure to sudden extreme negative market shifts.
\end{abstract}

Keywords: Asymmetric jumps, Portfolio diversification, Systematic risk, Equity JEL Classification: C58, G11, C61 


\section{Introduction}

The recent 2007-2008 and 2011 financial crises have revived a considerable degree of scepticism of portfolio theory. The availability of high-frequency data has led to great improvements in our ability to measure risk, allowing us to separate risk into its contributing factors. As a result, asset allocators are making changes in how they manage risk. Portfolio managers are becoming increasingly aware of the pitfalls of approaches that fail to address downside risk, or more specifically, extreme negative events. Risk factor diversification is becoming the focus. In this paper, we separate positive and negative jumps, using each family of jumps as risk factors, and analyse portfolio sensitivities to these risk factors as the number of portfolio holdings varies.

An important feature explored in our study is the asymmetry in portfolios' behaviour during extreme market downturns versus extreme market upsurges. Sudden large market shifts are rare events, but have substantially higher impacts than the diffusive price movements. Hedging against these extreme shifts is difficult, unless the portfolios are large enough to diversify away such risks. Recent studies by Bollerslev et al. (2008), Jacod and Todorov (2009) and Mancini and Gobbi (2012) have all argued for the presence of common jump arrivals across different assets, thus possibly inducing stronger dependencies in the "extreme". However, Bajgrowicz et al. (2016) argue that no co-jump affects all stocks simultaneously, suggesting jump risk is diversifiable. Our analysis shows stronger concordance between market and portfolio returns during extreme market downturns than during market upsurges, and reveals a large disparity in recommended number of portfolio holdings.

In evaluating the impact of extreme negative and positive market shifts on portfolios we investigate the extent the downside and upside jump risk can be diversified away. In particular, we address the following questions: How many stocks should investors hold on average, in order to reduce the sensitivity to market jumps to a certain level? How does the 
recommended portfolio size changes with the magnitude of extreme events? Are there any differences in recommended portfolio sizes for investors seeking to diversify against negative extreme events only? We find that more stocks are required to stabilise portfolio sensitivities to extreme negative market jumps than to extreme positive ones. ${ }^{1}$ In addition, the more we focus on the tails of distributions, the larger the difference we anticipate in their behaviours. When defining sudden extreme shifts, we explore several thresholds and consider the asymmetry effects for different levels of extreme market movements. We expect the difference in recommended portfolio sizes to be larger in the presence of more extreme jumps. Ignoring asymmetry results in under-diversification of portfolios and increases portfolio exposure to extreme negative market jumps.

Our analysis combines the developments from two strands of literature: modelling of extreme events and jump identification. Our contribution is, firstly, the evaluation of extreme negative vs extreme positive market shifts separately and, secondly, its impact in a portfolio setting. Some research has been done on the asymmetric tail risk (Ang et al., 2006; van Oordt and Zhou, 2016) ${ }^{2}$, including a companion paper by Alexeev et al. (2016) which only contrasts continuous and discontinuous systematic risks. In this paper, we use the inferential procedure of Li et al. (2017) to extend the single jump beta to the positive and negative jump betas. To the best of our knowledge no papers have investigated the behavior of signed systematic jump risk in a portfolio setting and the implications it would have in portfolio risk management and diversification to extreme market events.

\footnotetext{
${ }^{1}$ This is in line with the previous literature that shows that correlations among securities tend to increase during turbulent market conditions, making it harder to diversify portfolios. The increase in correlation coefficients during periods of distress is well recognized in the literature (see, for example, Forbes and Rigobon, 2002; Fry et al., 2010; Rigobon, 2016).

${ }^{2}$ van Oordt and Zhou (2016) provide evidence that historical tail betas do capture future systematic risk pointing to the empirically persistent tail betas. They show that stocks with historically high tail betas suffer losses during future market crashes that are on average 2 to 3 times larger than their low-tail-beta counterparts. Although the argument underlying the persistency of tail betas is cogent, in our analysis we utilise 5-minute instead of daily data.
} 
In the past two decades, modelling extreme events has become mainstream in risk management practice. ${ }^{3}$ Regulators are attentive to market conditions during a crisis because they are concerned with the protection of the financial system against catastrophic events. Bates (2008) formalises the intuition that investors treat extreme events differently than they treat more common and frequent ones. The increased availability of high-frequency data amplified the interest in the analysis of these tail events. ${ }^{4}$ Modelling rare and extreme events often explains the high observed equity risk premia by taking into account the premia for rare events, provided that these events are sufficiently severe (Rietz, 1988; Barro, 2006; Bates, 2008). ${ }^{5}$

The jumps in the high-frequency literature may be rare events when considered spatially, but often appear too frequently in calendar time to be considered extreme or disastrous. The need for a refined classification of jumps according to their magnitude and its association with extreme events is apparent. Mounting empirical evidence in the high-frequency literature suggests that jumps occur on $4 \%-13 \%$ of days per year on average (e.g., Andersen et al., 2007; Patton and Verardo, 2012; Alexeev et al., 2017, among others). It can be argued, however, that events that occur this frequently can hardly be classified as "extreme". ${ }^{6}$ The jump identification literature offers a number of methods that can sieve out less extreme

\footnotetext{
${ }^{3}$ Francois Longin, program chair of ESSEC Business School Conference on Extreme Events in Finance held on December 15-17, 2014, writes: "When I started to study extreme events in finance after the stock market crash of October 1987, academic studies considered extreme events as outliers and such data were usually discarded from empirical work. A few decades later I am more than happy to organize an international conference on extreme events in finance." (http://extreme-events-finance.net/scientific-committee/). Extreme value theory and tail behaviour, as well as its applications in finance and insurance, are extensively discussed in Embrechts et al. (1997).

${ }^{4}$ For example, Bollerslev and Todorov (2011) develop a new framework for estimating jump tails, and, using 1-minute data, find strong evidence for richer and more complex dynamic dependencies in the jump tails than previously entertained in the literature.

${ }^{5}$ Another plausible explanation of the equity premium puzzle could be that investors tend to overweight the probability of rare, extreme events. This probability weighting can independently generate a large equity premium (Giorgi and Legg, 2012; Barberis, 2013).

${ }^{6}$ Christensen et al. (2014), using ultra-high frequency data, argue that jumps in financial asset prices are often erroneously identified and are not nearly as common as generally thought.
} 
events by varying the threshold used in its detection (e.g., Mancini, 2001; Mancini and Renò, 2011; Davies and Tauchen, 2015). Thus, in our empirical application, in addition to threshold levels commonly used in the jump identification literature, we allow for the thresholds high enough to investigate the most severe occasions only. In line with Christensen et al. (2014), we detect far fewer jumps than what is usually found in the literature. More importantly, it is only at the higher jump detection threshold that we begin to observe the asymmetry in portfolio sensitivities to market jumps.

This asymmetry has crucial implications for portfolio allocation decisions. Investors typically perceive downside and upside extreme events differently. ${ }^{7}$ It is believed that the fear of large negative shocks is a component that drives asset prices, because investors expect compensation for the risk that such a rare event occurs. It is not only the occurrence of rare events but also the very fear of them that influences investors' behaviour and market prices. Bollerslev and Todorov (2011) explore pricing implications for jump risk in periods of extreme downside losses as opposed to extreme upside gains, and check if investors demand additional compensation for holding stocks with high sensitivities to these movements. Results show that, although the behaviour of the two tails is clearly related, the contributions to the overall risk premium are far from symmetric. Further evidence on asymmetric effects of jump risk measures can be found in Guo et al. (2015) and Audrino and Hu (2016). These findings highlight the importance of considering the asymmetry effects of extreme events in portfolio risk management.

\footnotetext{
${ }^{7}$ It has long been recognised that investors care differently about downside losses versus upside gains. Ang et al. (2006) show that the cross section of stock returns reflects a downside risk premium of $6 \%$ per annum. The reward for bearing downside risk is not simply compensation for regular market beta, nor it is explained by common stock market return predictors. Bollerslev and Todorov (2011); Audrino and Hu (2016) find similar results but for downside and upside extreme events. The economic intuition underlying downside risk is simple: Agents require a premium not only for securities the more their returns co-vary with the market return, but also, and even more so, when securities co-vary more with market returns conditional on low market returns.
} 
In this paper, we provide equity portfolio size recommendations to stabilize portfolio jump betas. We find that the number of stocks required to stabilise portfolio exposure to sudden large negative price changes in the market is substantially greater than under a scenario where only positive jumps are considered, or when the asymmetry is not taken into account. We show that correlations between extreme market returns and corresponding stock returns increases during crises years, and are more pronounced for negative jumps. Thus, for a naive portfolio, using conservative conjecture on how many stocks to hold (i.e., the maximum number of recommended holdings across jump types, and threshold levels), we recommend at least 54 holdings. For example, based on portfolio holdings data from the Center for Research in Security Prices (CRSP), the interquantile range of the number of holdings for domestic equity mutual funds is $44-132$ with median of 75 holdings. Consequently, funds with less than 54 holdings, representing 35 percent of all equity mutual funds, are unnecessarily exposed to extreme market drops.

The remainder of the paper is organised as follows. Section II sets up the model framework. The data used in our empirical investigation are detailed in Section III. We investigate the behaviours of systematic negative and positive jump risk factors in portfolios of assets in Section V. Section VI concludes.

\section{Model Setup}

We start with a panel of $N$ assets over a fixed time interval $[0, T]$. Following the convention in the high-frequency financial econometrics literature, we assume the log-price $p_{i, t}$ of the $i^{\text {th }}$ asset follows a semi-martingale plus jumps process in continuous time. In turn, the log-return of any asset, $r_{i, t}$, has the following representation:

$$
r_{i, t} \equiv \mathrm{d} p_{i, t}=b_{i, t} \mathrm{~d} t+\sigma_{i, t} \mathrm{~d} W_{i, t}+\kappa_{i, t} \mathrm{~d} \mu_{i, t}, \quad t \in[0, T], \quad i=1,2, \ldots, N,
$$


where $b_{i, t}$ is a locally bounded drift term, $\sigma_{i, t}$ denotes the non-zero spot volatility, $W_{i, t}$ is a standard Brownian motion for asset $i$. The $\kappa_{i, t} \mathrm{~d} \mu_{i, t}$ term represents the jump component. The jump measure $\mathrm{d} \mu_{i, t}$ is such that $\mathrm{d} \mu_{i, t}=1$ if there is a jump in $r_{i, t}$ at time $t$, and $\mathrm{d} \mu_{i, t}=0$ otherwise. The size of jump at time $t$ is represented by $\kappa_{i, t}$. In fact, $\kappa_{i, t}$ can be defined as $\kappa_{i, t}=p_{i, t}-p_{i, t-}$ in general, where $p_{i, t-}=\lim _{s \uparrow t} p_{i, s}$ for $s$ increasing in value and approaching $t$. It follows immediately that $\kappa_{i, t}=0$ for $t \in\left\{t: \mathrm{d} \mu_{i, t}=0\right\}$ under this definition, meaning that at times when jump does not occur, the size of jump is zero.

Return on the market portfolio $r_{0, t}$ can be decomposed in a way similar to (1):

$$
r_{0, t}=b_{0, t} \mathrm{~d} t+\sigma_{0, t} \mathrm{~d} W_{0, t}+\kappa_{0, t} \mathrm{~d} \mu_{0, t}
$$

We assume that the jumps in processes (1) and (2) have only finite activity. Processes with finite activity jumps, as opposed to the infinite activity, have only a finite number of jumps in $[0, T]$. Since we only focus on "big" jumps with sizes bounded away from zero, this assumption is not overly restrictive. For a detailed discussion on finite versus infinite activity in jumps see Ait-Sahalia and Jacod (2012) among others.

\section{A. Jump Regression}

In studying jump dependence between equity portfolios and the broad market index, we use high-frequency observations focusing on segments of data on the fringes of return distributions. Thus, we only consider a few outlying observations that, at the time, are informative for the jump inference. In particular, we study the relationship between jumps of a process for a portfolio of assets and an aggregate market factor, and we analyse the comovement of the jumps in these two processes. By increasing the threshold used to identify jumps, we focus on the most pronounced (extreme) events. For instance, we present in Figure 1 the frequency of positive and negative jump occurrence for each year from 2003 to 
2011. For more liberal truncation thresholds, more than 100 jumps can be identified in most years.

We study the dependence between the jump components of individual assets (or portfolios) and that of the market return, utilising the methodology proposed by Li et al. (2015, 2017). Let $\mathcal{T}$ be the collection of jump times for the market portfolio $r_{0, t}$, i.e. $\mathcal{T}=\{\tau$ : $\left.\mathrm{d} \mu_{0, \tau}=1, \tau \in[0, T]\right\}$. The set $\mathcal{T}$ has finite elements almost surely given the assumption of finite activity jumps. Given the predominance of factor models in asset pricing applications, we use a linear relationship to assess the sensitivity of jumps in portfolios to jumps in the market. In parallel with the classical one-factor market model, we set the linear factor model for jumps in the following form

$$
\kappa_{i, \tau}=\beta_{i}^{d} \kappa_{0, \tau}+\epsilon_{i, \tau}, \quad \tau \in \mathcal{T}, \quad i=1,2, \ldots, N
$$

where the superscript $d$ stands for discontinuous (or jump) beta, and $\epsilon_{i, \tau}$ is the residual series. We only consider the jump times of the market portfolio $\mathcal{T}$ because $\beta_{i}^{d}$ is not identified elsewhere. Therefore, $\beta_{i}^{d}$ only exists if there is at least one jump in $r_{0, t}$ in $[0, T]$. Model (3) implicitly assumes that $\beta_{i}^{d}$ is constant over the interval $[0, T]$.

The jump beta $\beta_{i}^{d}$ in (3) has a similar interpretation to the market beta in the CAPM model. It allows us to assess the sensitivity of an asset (or a portfolio of assets) to extreme market fluctuations. Lower $\beta_{i}^{d}$ would signify a resistance of an asset or portfolio to move as much as a market during extreme event (jump defensive assets), while higher $\beta_{i}^{d}$ values represent high sensitivity of an asset exacerbating the effect of the market moves during the extreme event (jump sensitive assets). 


\section{B. Isolating Jumps from the Brownian Component}

Under discrete-time sampling, neither the jump times $\mathcal{T}$ nor jump sizes $\kappa_{i, \tau}$ are directly observable from the data. Suppose the price and return series are observed every $\Delta$ interval, i.e. we obtain return series $r_{i, \Delta}, r_{i, 2 \Delta}, \ldots, r_{i, m \Delta}$, where $m=\lfloor T / \Delta\rfloor$, for $i=0,1, \ldots, N$.

Our first step in constructing the jump regression model (3) is to identify the discrete-time returns on the market portfolio $r_{0, j \Delta}=p_{0, j \Delta}-p_{0,(j-1) \Delta}$ that contain jumps, $j=1,2, \ldots, m$. We use the truncation threshold proposed by Mancini (2001) for this purpose (see also Mancini, 2009; Mancini and Renò, 2011). The threshold, denoted by $u_{0, m}$, is a function of the sampling interval $\Delta$, and hence the sampling frequency $m$. The most widely used threshold is

$$
u_{0, m}=\alpha \Delta^{\omega}, \quad \text { with } \quad \alpha>0 \text { and } \omega \in(0,1 / 2)
$$

Taking into account the time-varying spot volatility of the return series, the constant $\alpha$ is usually different for different assets, and could vary over time (see, for example, Jacod, 2008). One example is to set $\alpha$ to be dependent on the estimated continuous volatility of the given asset.

As $\Delta \rightarrow 0$ and $m \rightarrow \infty$, the condition $\left|r_{0, j \Delta}\right|>u_{0, m}=\alpha \Delta^{\omega}$ eliminates the continuous diffusive returns on the market portfolio asymptotically, and hence only keeps returns that contain jumps. We collect the indices of these discrete-time intervals where the market return exceeds the truncation level, and denote this set as

$$
\mathcal{J}_{m}=\left\{j: 1 \leq j \leq m,\left|r_{0, j \Delta}\right|>u_{0, m}\right\}
$$

We denote the collection of interval returns for $\mathcal{J}_{m}$ by $\left\{r_{0, j \Delta}\right\}_{j \in \mathcal{J}_{m}}$. Correspondingly, in the continuous-time data generating process for market return (2), for each jump time $\tau \in \mathcal{T}$, 
we also find the index $j$ such that the jump $\kappa_{0, \tau}$ occurs in the interval $((j-1) \Delta, j \Delta]$,

$$
\mathcal{J}=\{j: 1 \leq j \leq m, \tau \in((j-1) \Delta, j \Delta] \text { for } \tau \in \mathcal{T}\}
$$

An important result from Li et al. (2017) is that, under some general regularity conditions, the probability that the set $\mathcal{J}_{m}$ coincides with $\mathcal{J}$ converges to one as $\Delta \rightarrow 0$. This is formally stated in Proposition 1 in Li et al. (2017) that we recall for convenience.

Proposition 1. Under certain regularity assumptions, as $\Delta \rightarrow 0$, we have

(a) $\mathbb{P}\left(\mathcal{J}_{m}=\mathcal{J}\right) \rightarrow 1$

(b) $\left((j-1) \Delta, r_{0, j \Delta}\right)_{j \in \mathcal{J}_{m}} \stackrel{\mathbb{P}}{\longrightarrow}\left(\tau, \kappa_{0, \tau}\right)_{\tau \in \mathcal{T}}$.

Note that Proposition 1(a) implies that the number of elements in the set $\mathcal{J}_{m}$ consistently estimates the number of jumps in the process $r_{0, t}$; while $1(\mathrm{~b})$ states that as $\Delta \rightarrow 0$, the starting point of the interval $(j-1) \Delta$, consistently estimates the jump time $\tau$, and the interval return $r_{0, j \Delta}$ consistently estimates the jump size $\kappa_{0, \tau}$. The asymptotic results in Proposition 1 provides a powerful tool of linking the discrete-time return observations to the unobservable jumps and jump times in the continuous time. We use the discrete-time return observations to estimate the jump regression (3) and obtain consistent estimator of the jump beta $\beta_{i}^{d} .8$

\section{Estimating Jump Beta}

In accordance with model (3), the discrete-time jump regression model has the form

$$
r_{i, j \Delta}=\beta_{i}^{d} r_{0, j \Delta}+\epsilon_{i, \tau}, \quad j \in \mathcal{J}_{m}, \quad i=1,2, \ldots, N
$$

\footnotetext{
${ }^{8}$ In our analysis we focus on the 5-min frequency but we evaluate our results for a range of frequencies. To minimise the effect of microstructure noise, we also apply pre-averaging method proposed in Mykland and Zhang (2016). See Alexeev et al. (2016) for details.
} 
Hence a naïve consistent estimator of $\beta_{i}^{d}$ is the analog of the OLS estimator,

$$
\tilde{\beta}_{i}^{d}=\frac{\sum_{j \in \mathcal{J}_{m}} r_{i, j \Delta} \cdot r_{0, j \Delta}}{\sum_{j \in \mathcal{J}_{m}}\left(r_{0, j \Delta}\right)^{2}}, \quad i=1,2, \ldots, N
$$

Li et al. (2017) propose an efficient estimator for $\beta_{i}^{d}$ in (7). It is an optimal weighted estimator in the sense that it minimizes the conditional asymptotic variance among all weighting schemes. The optimal weight $w_{j}^{*}$ is a function of the preliminary consistent estimator $\tilde{\beta}_{i}^{d}$, and the approximated pre-jump and post-jump spot covariance matrices $\hat{C}_{j-}$ and $\hat{C}_{j+}$ :

$$
w_{j}^{*}=\frac{2}{\left(-\tilde{\beta}_{i}^{d}, 1\right)\left(\hat{C}_{j-}+\hat{C}_{j+}\right)\left(-\tilde{\beta}_{i}^{d}, 1\right)^{\prime}}, \quad \text { for } \quad j \in \overline{\mathcal{J}}_{m}
$$

where $\overline{\mathcal{J}}_{m}=\left\{j \in \mathcal{J}_{m}: k_{m}+1 \leq j \leq m-k_{m}\right\}$, and $k_{m}$ is an integer such that $k_{m} \rightarrow \infty$ and $k_{m} \cdot \Delta \rightarrow 0$ as $\Delta \rightarrow 0$. The spot covariance matrices are estimated in the following manner. We construct the truncation threshold for the vector $\boldsymbol{r}_{j \Delta} \equiv\left(r_{0, j \Delta}, r_{i, j \Delta}\right)$ jointly in the same way as in (4), and denote it as $\boldsymbol{u}_{m} \equiv\left(u_{0, m}, u_{i, m}\right)$. For any $j \in \overline{\mathcal{J}}_{m}$, we have

$$
\begin{aligned}
& \hat{C}_{j-}=\frac{1}{k_{m} \cdot \Delta} \sum_{l=1}^{k_{m}} \boldsymbol{r}_{\left(j+l-k_{m}-1\right) \Delta}^{\prime} \cdot \boldsymbol{r}_{\left(j+l-k_{m}-1\right) \Delta} \cdot \mathbb{1}_{\left\{\left|\boldsymbol{r}_{\left(j+l-k_{m}-1\right) \Delta}\right| \leq \boldsymbol{u}_{m}\right\}}, \\
& \hat{C}_{j+}=\frac{1}{k_{m} \cdot \Delta} \sum_{l=1}^{k_{m}} \boldsymbol{r}_{(j+l) \Delta}^{\prime} \cdot \boldsymbol{r}_{(j+l) \Delta} \cdot \mathbb{1}_{\left\{\left|\boldsymbol{r}_{(j+l) \Delta}\right| \leq \boldsymbol{u}_{m}\right\}},
\end{aligned}
$$

as the approximated pre-jump and post-jump spot covariance matrices, respectively.

Given any weighting function $w_{j}$, the class of weighted estimators $\hat{\beta}_{j}^{d}$ can be represented as

$$
\hat{\beta}_{i}^{d}=\frac{\sum_{j \in \overline{\mathcal{J}}_{m}} w_{j} \cdot r_{i, j \Delta} \cdot r_{0, j \Delta}}{\sum_{j \in \overline{\mathcal{J}}_{m}} w_{j} \cdot\left(r_{0, j \Delta}\right)^{2}}, \quad i=1,2, \ldots, N
$$

In Theorem 2, Li et al. (2017) show that the weighting function in (9) combined with the estimator (12) produces the most efficient estimate of the jump beta $\beta_{i}^{d}$. The standard errors 
and subsequently the confidence intervals of the estimators can be constructed using the bootstrap procedure outlined in Li et al. (2015). However, the authors restrict the negative and positive betas to be the same. In the next section, we consider the positive jump and negative jump separately and allow for the difference in impact of the two tail activities.

\section{Asymmetric Jump Beta}

In developing Modern Portfolio Theory in 1959, Henry Markowitz recognized that since only downside deviation is relevant to investors, using downside deviation to measure risk would be more appropriate than using standard deviation (Markowitz, 1971). Ang et al. (2006) explore the asset pricing implications of the downside risk without, however, separately considering extreme events or jumps. In this section, instead of pooling jumps at both positive and negative ends together, we examine the jump covariation between individual asset (or portfolio) and the market index at times of positive market jumps and negative market jumps separately.

Although we focus on the negative jump in the market portfolio and the negative jump beta associated with it, our modelling approach naturally gives rise to a similar definition of the positive jump beta. The naive estimators of the two asymmetric betas $\tilde{\beta}_{i}^{d+}$ and $\tilde{\beta}_{i}^{d-}$ are as follows:

$$
\begin{aligned}
& \tilde{\beta}_{i}^{d-}=\frac{\sum_{j \in \mathcal{J}_{m}} r_{i, j \Delta} \cdot r_{0, j \Delta} \cdot \mathbb{1}_{\left\{r_{0, j \Delta}<0\right\}}}{\sum_{j \in \mathcal{J}_{m}}\left(r_{0, j \Delta}\right)^{2} \cdot \mathbb{1}_{\left\{r_{0, j \Delta}<0\right\}}}, \\
& \tilde{\beta}_{i}^{d+}=\frac{\sum_{j \in \mathcal{J}_{m}} r_{i, j \Delta} \cdot r_{0, j \Delta} \cdot \mathbb{1}_{\left\{r_{0, j \Delta}>0\right\}}}{\sum_{j \in \mathcal{J}_{m}}\left(r_{0, j \Delta}\right)^{2} \cdot \mathbb{1}_{\left\{r_{0, j \Delta}>0\right\}}},
\end{aligned}
$$

for $i=1,2, \ldots, N$. When calculating the weighted estimators, the weighting function (9) 
would differ for the positive and negative jump betas:

$$
\begin{aligned}
& w_{j}^{-}=\frac{2}{\left(-\tilde{\beta}_{i}^{d-}, 1\right)\left(\hat{C}_{j-}+\hat{C}_{j+}\right)\left(-\tilde{\beta}_{i}^{d-}, 1\right)^{\prime}}, \quad \text { for } \quad j \in \overline{\mathcal{J}}_{m} \text { and } r_{0, j \Delta}<0, \\
& w_{j}^{+}=\frac{2}{\left(-\tilde{\beta}_{i}^{d+}, 1\right)\left(\hat{C}_{j-}+\hat{C}_{j+}\right)\left(-\tilde{\beta}_{i}^{d+}, 1\right)^{\prime}}, \quad \text { for } \quad j \in \overline{\mathcal{J}}_{m} \text { and } \quad r_{0, j \Delta}>0 .
\end{aligned}
$$

Here we assume that before and after the jumps, the spot covariance matrices are the same for positive and negative jumps. These lead to the formation of the weighted estimators of the asymmetric betas:

$$
\begin{aligned}
& \hat{\beta}_{j}^{d-}=\frac{\sum_{j \in \overline{\mathcal{J}}_{m}} w_{j}^{-} \cdot r_{i, j \Delta} \cdot r_{0, j \Delta} \cdot \mathbb{1}_{\left\{r_{0, j}<0\right\}}}{\sum_{j \in \overline{\mathcal{J}}_{m}} w_{j}^{-} \cdot\left(r_{0, j \Delta}\right)^{2} \cdot \mathbb{1}_{\left\{r_{0, j}<0\right\}}}, \\
& \hat{\beta}_{j}^{d+}=\frac{\sum_{j \in \overline{\mathcal{J}}_{m}} w_{j}^{+} \cdot r_{i, j \Delta} \cdot r_{0, j \Delta} \cdot \mathbb{1}_{\left\{r_{0, j \Delta}>0\right\}}}{\sum_{j \in \overline{\mathcal{J}}_{m}} w_{j}^{+} \cdot\left(r_{0, j \Delta}\right)^{2} \cdot \mathbb{1}_{\left\{r_{0, j}>0\right\}}},
\end{aligned}
$$

for $i=1,2, \ldots, N$.

In what follows, we will use real data to test the asymmetry in the jump betas and its implications for portfolio risk management.

\section{Data}

We investigate the behavior of the $\beta_{i}^{d+}$ and $\beta_{i}^{d-}$ estimates over the period from January 2, 2003 to December 30, 2011. This period includes the financial crisis associated with the bankruptcy of Lehman Brothers in September 2008 and the subsequent period of turmoil in US and international financial markets. The underlying data are tick-by-tick price observations on 501 stocks drawn from the constituent list of the S\&P500 index during our sample period, obtained from Thomson Reuters Tick History through Securities Industry Research Centre of Asia-Pacific (SIRCA). The constituent list is constructed similar to Dungey et al. (2012) and does not intend to cover all stocks listed on the S\&P500 index. We only include 
stocks with sufficient coverage and data availability for high-frequency time series analysis of this type.

\section{A. Data Processing and Preparation}

The original dataset consists of over 900 stocks taken from the 0\#.SPX mnemonic Reuters Identification Code (RIC) code for the S\&P500 index historical constituents. This included a number of stocks which trade OTC and on alternative exchanges, as well as some which altered currency of trade during the period; these stocks were excluded. We adjusted the dataset for changes in RIC codes during the sample period to account for mergers and acquisitions, stock splits, and trading halts. We also removed stocks with insufficient observations during the sample period. The data handling process is fully documented in the web-appendix to Dungey et al. (2012). The final data set contains 501 individual stocks, hence $N=501$.

Tick-by-tick data are converted to 5-minute equidistant mesh using previous tick method (e.g., interval observations recorded as the last trade within the interval). ${ }^{9}$ The 5 -minute sampling frequency is chosen as relatively conventional in the high-frequency literature, especially for univariate estimation (see, for example, Andersen et al., 2007; Lahaye et al., 2011; Liu et al., 2015). Optimal sampling frequency is an area of ongoing research, and despite the univariate work by Bandi and Russell (2006), this issue is highly contentious, especially when analyzing multiple series with varying degrees of liquidity. The 5-minute frequency is much finer than those employed in Todorov and Bollerslev (2010); Bollerslev et al. (2008), both of which use 22.5-minute data. Lower sampling frequencies are generally employed due to concerns over the Epps effect (Epps, 1979); however, as the quality of highfrequency data and market liquidity have improved in many ways, finer sampling does not

\footnotetext{
${ }^{9}$ Originally proposed by Wasserfallen and Zimmermann (1985), the method remains the most common sampling scheme in the empirical high frequency literature.
} 
threaten the robustness of our results. ${ }^{10}$

Our analysis of tick-by-tick data using previous tick sampling scheme, show that the range of frequencies from 90-second to 600-second produce stable jump regression results. ${ }^{11}$ With regards to the sampling scheme we refer to Alexeev et al. (2017) where the authors contrast the effect of different frequencies on jump detection using pre-averaging scheme of Mykland and Zhang (2016) and the previous tick method. The findings suggest that, although the pre-averaging of the data helps mitigate the effect of microstructure noise at higher frequencies, it may inadvertently pulverise jumps and lead to lower detection rates.

The intra-day price and return data start at 9:30 am and end at 4:00 pm, observations with time stamps outside this window and overnight returns are removed. Missing 5-minute price observations are filled forward resulting, in such cases, in zero inter-interval returns. In the case where the first observations of the day are missing, we use the first non-zero price observation on that day to fill backwards. "Bounce back" outliers as defined in Aït-Sahalia et al. (2011) are also removed. Thus, we have 77 intra-day observations for 2262 trading days. Estimates of $\beta_{i}^{d+}$ and $\beta_{i}^{d-}$ are computed on an annual basis. High-frequency data permits the use of 1-year non-overlapping windows to analyse the dynamics of our systematic risk estimates. Li et al. (2017) also find in their empirical application using US equity market data that the jump beta remains constant over a year most of the time. Given the 5-minute sampling frequency, not all stocks in the S\&P 500 list have sufficient data coverage to perform the analysis. Thus, each year, we consider a subset of stocks that have at least $75 \%$ of

\footnotetext{
${ }^{10}$ Investigating continuous and discontinuous betas in the cross section of expected returns, Bollerslev et al. (2016) favour a 75-minute sampling frequency to overcome the lack of liquidity across their sample of stocks, whilst we use a 5-minute sampling frequency which gives us better properties for the in-fill asymptotics for the statistical procedures we employ. We conducted Monte Carlo experiments demonstrating the small sample properties to support our contention that the statistics work well in our scenario. In addition, we investigated the stability of our continuous and discontinuous beta estimates using a range of sampling frequencies from 1 second to 1,800 seconds.

${ }^{11}$ Based on signature plots similar to Figure 2 later in the paper.
} 
the entire 1-year window as non-zero 5-min return data. Depending on the year, this filter removes 30-40 stocks from the 501 available constituents. We construct an equally weighted portfolio of all remaining stocks in each estimation window as the benchmark market portfolio. We side with Bollerslev et al. (2008) and use equally weighted portfolios rather than value weighted ones to avoid situations where the weight on one stock is disproportionately large relative to other portfolio constituents. This issue will become especially pertinent in the portfolio simulation section later in the paper. ${ }^{12}$

\section{B. Parameter Values}

In our empirical application we normalize each trading day to be one unit in time. Given the number of observations in each day $m=77$, the sampling frequency is $\Delta=1 / 77$.

Parameters in the truncation threshold (4) are chosen as follows. The constant $\omega=0.49 .{ }^{13}$ Taking into account the time-varying volatility $\sigma_{i, t}$, we set $\alpha$ to be a function of the estimated daily continuous volatility component for each individual asset. In finite sampling, the continuous volatility is consistently estimated by the bipower variation (Barndorff-Nielsen and Shephard, 2004, 2006):

$$
B V_{i}=\left(\frac{\pi}{2}\right) \cdot \sum_{j=1}^{m-1}\left|r_{i, j \Delta}\right|\left|r_{i,(j+1) \Delta}\right| \stackrel{\mathbb{P}}{\longrightarrow} \int_{0}^{T} \sigma_{i, t}^{2} \mathrm{~d} t \quad \text { as } \quad \Delta \rightarrow 0, \quad i=0,1, \ldots, N
$$

We set $\alpha_{i}=a \sqrt{B V_{i}}$, where $a=3,4,5$. This leads to the threshold $u_{i, m}=a \sqrt{B V_{i}} \cdot(1 / 77)^{0.49}$. The choice of using a multiple of the estimated continuous volatility is relatively standard in the literature for disentangling jumps from the continuous price movements. It serves the purpose of controlling for the possibly time-varying spot volatility automatically in jump

\footnotetext{
${ }^{12}$ See Fisher (1966) for the discussion of "Fisher's Arithmetic Index", an equally weighted average of the returns on all listed stocks.

${ }^{13}$ In most empirical literature, typical values for $\mathcal{\omega}$ are between 0.45 and 0.49 . See Jacod and Protter (2012, p. 248) for a discussion.
} 
detection. In our empirical application, $B V_{i}$ is calculated daily ensuing a different threshold for each trading day. ${ }^{14}$ We find both positive and negative jumps in each estimation window (year) in the market portfolio, and hence $\beta_{i}^{d+}$ and $\beta_{i}^{d-}$ can be estimated for each year. Since the main aim of the paper is to identify the consequence of ignoring asymmetries in jump betas, we use the same threshold independent of the sign of the jump. Moreover, the BV formula in (19) is ambiguous for semi-BV estimation - splitting BV for negative and positive returns - since it relies on two consecutive returns allowing for four possible scenarios rather than only two as in the realized semi-variance (Barndorff-Nielsen et al., 2010) or the downward absolute power variation (Visser, 2008).

\section{Empirical Analysis}

In this section we analyse the statistical properties of betas estimated based on the overall market returns, and based on the upside and downside market returns separately.

\section{A. Market Volatility and Jumps}

Figure 1 plots the square root of the daily bipower variation of the equally weighted market index on the left axis (black line), and the number of positive (blue) and negative (red) jumps for each year in the 2003-2011 sample period on the right axis. The subsample before mid-2007 is much less volatile than the second half of the sample which includes the global financial crisis (GFC). Market volatility has increased considerably since mid-2007, which is usually regarded as the initial emergence of the GFC, and peaked in late 2008

\footnotetext{
${ }^{14}$ Alternatively, for data at higher sampling frequencies, the threshold can be estimated on hourly basis to incorporate the intra-day volatility pattern which might make the identification of jumps more efficient. Andersen et al. (2012) analyse diurnal patterns in a sample of DJ30 stocks and observe the U-shape intra-day volatility pattern with volatility clearly elevated during the first hour of trading. This may be attributed to the release of regularly scheduled macroeconomic announcements at 7:30am and 9:00am CT. Given that our focus is on the S\&P 500 constituents where not all stocks are as heavily traded as the ones in the DJ30 and on that we employ the 5-minute sampling frequency, daily $B V_{i}$ s are calculated omitting the first hour of trading. For a detailed discussion on adaptive truncation which endogenously controls for the local level of volatility refer to Andersen et al. (2012).
} 
Figure 1: Daily bipower variation of equally weighted market index (left axis) versus the identified number of positive (blue) and negative (red) jumps (right axis). Jumps where identified using the following thresholds: $\alpha_{i}=3 \sqrt{B V_{i}}$ (top panel), $\alpha_{i}=4 \sqrt{B V_{i}}$ (middle panel) and $\alpha_{i}=5 \sqrt{B V_{i}}$ (bottom panel).
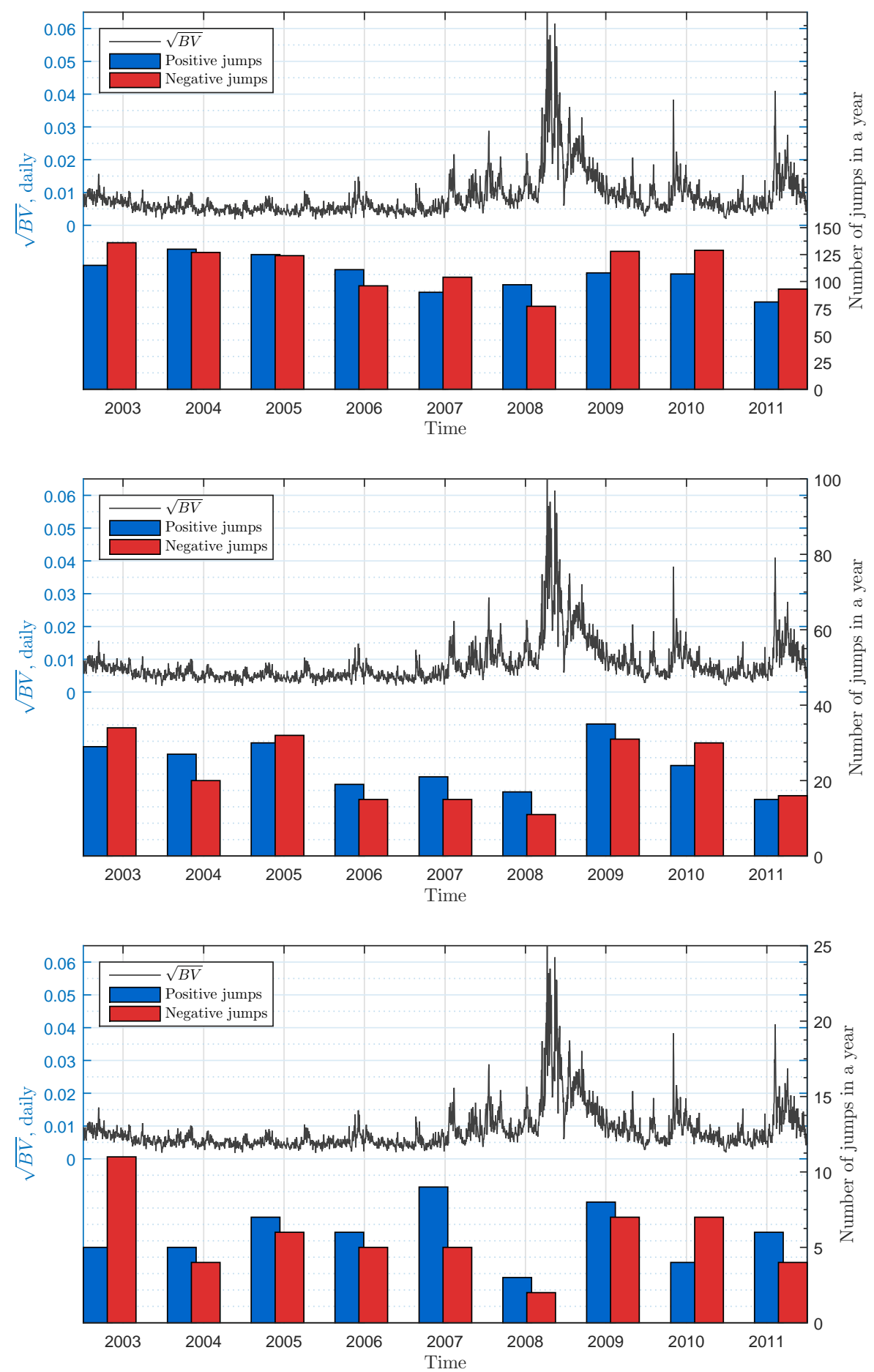
during the few months after the bankruptcy of Lehman Brothers, the bailout of AIG and the announcement of the TARP (Troubled Asset Relief Program). Other highly volatile periods include mid-2010 during the Greek debt crisis, and late 2011 during the European sovereign debt crisis with the deterioration of economic conditions in the Eurozone as a whole. The two peak values of market volatility after the GFC correspond to the May 6, 2010 flash crash and August 9, 2011. On August 5, 2011 Standard \& Poor's downgraded America's credit rating for the first time in history, followed with short-selling ban by Greece on August 8, 2011, and other 4 EU countries on August 11, 2011.

The choice of the threshold level in jump identification has important implications for our analysis. Lower thresholds generate too many jumps. For example, for threshold $\alpha_{i}=$ $3 \sqrt{B V_{i}}$, the number of negative and positive jumps identified often exceeds 100 per year, which implies that the jumps occur almost every other day. Jumps are rare events and should not happen that often. The number of jumps identified using thresholds $\alpha_{i}=4 \sqrt{B V_{i}}$ and $\alpha_{i}=5 \sqrt{B V_{i}}$ appear more realistic. ${ }^{15}$ Notably, Figure 1 shows that the volatile period during the GFC corresponds to fewer jumps in both directions. In particular, in 2008, using the most conservative threshold, $\alpha_{i}=5 \sqrt{B V_{i}}$, we observe fewer than 5 jumps (positive or negative). This result is expected as the market volatility is generally higher during crisis than during calmer period, the threshold of detecting jump observations will be elevated accordingly to prevent mistaking volatility bursts from real jumps. Li et al. (2017), analysing the period from 2007 to 2012, identify 2008 as the year with the smallest number of jumps. Black et al. (2012) also observe that the stock market has fewer jumps during crisis periods. Since only the jump observations are taken to estimate the non-weighted asymmetric beta in (13) and (14), low number of observations could certainly affect the quality of the estimates. Hence, it is necessary to use the weighted estimators, (17) and (18), in order to reduce the

\footnotetext{
${ }^{15}$ When using $\alpha_{i}=6 \sqrt{B V_{i}}$ as our threshold, we failed to identify any jumps in a number of years.
} 
small sample size effect.

\section{B. Estimation Results}

In addition to the weighted estimators of the overall jump beta (12) and the asymmetric betas (17) and (18), we also calculate the continuous beta obtained using the OLS regression in the spirit of Andersen et al. (2006)'s realized beta

$$
\hat{\beta}_{i}^{c}=\frac{\sum_{j=1}^{m} r_{i, j \Delta} \cdot r_{0, j \Delta} \cdot \mathbb{1}_{\left\{\left|r_{0, j \Delta}\right| \leq u_{0, m}\right\}}}{\sum_{j=1}^{m}\left(r_{0, j \Delta}\right)^{2} \cdot \mathbb{1}_{\left\{\left|r_{0, j \Delta}\right| \leq u_{0, m}\right\}}}, \quad i=1,2, \ldots, N,
$$

where, in contrast, only the 5-minute return observations below the threshold are used to construct $\hat{\beta}_{i}^{c}$. The continuous beta would be able to incorporate the impact of co-movements in the continuous component of individual asset (or portfolio) and the market index.

In Table 1 we present the descriptive statistics and the correlations between the beta estimates as well as the average monthly excess returns of each asset. The means of all four estimated beta measures are very close to one. The distributions of the estimates are positively skewed with exception of $\beta^{d+}$. The average continuous beta is statistically different from zero, whereas the three average jump beta estimates are insignificant, possibly due to the high heterogeneity in jump sensitivities among assets. Monthly excess stock returns are very dispersed and negatively skewed.

We examine how much variation in individual stock returns could be explained by the jump component of the market return. Table 2 presents the average $R^{2}$ across all stocks using different thresholds for jump identification. The jump betas are calculated for each year separately. ${ }^{16}$ For the negative beta $\beta^{d-}$, the average $R^{2}$ increases with the threshold in

\footnotetext{
${ }^{16}$ On one hand, the jump regression fits can be further stabilised when the regressions are run over a shorter period, consistent with the conditional asset pricing models in which betas change over time. On the other hand, given the scarcity of more extreme events, sample size inevitably becomes an issue as periods get shorter. We follow Li et al. (2017) in setting the estimation period length of 1 year.
} 
Table 1: Summary Statistics $\ddagger$

\begin{tabular}{lccccc}
\hline \hline \multicolumn{2}{c}{$\hat{\beta}^{c}$} & $\hat{\beta}^{d}$ & $\hat{\beta}^{d+}$ & $\hat{\beta}^{d-}$ & $\bar{r}(\%)$ \\
\hline \multicolumn{2}{l}{ Panel A: Descriptive Statistics } & & & & \\
\hline $25 \%$ & 0.7576 & 0.5007 & 0.3155 & 0.2744 & -4.8019 \\
Mean & 0.9966 & 0.9882 & 0.9957 & 0.9933 & 0.2817 \\
$75 \%$ & 1.1828 & 1.3848 & 1.5476 & 1.6067 & 5.9589 \\
Std.Dev. & 0.3464 & 0.8910 & 2.1804 & 2.1674 & 12.9613 \\
Skew. & 0.8650 & 0.9346 & -0.4632 & 0.7260 & -168.9742 \\
\hline Panel B: Correlation Table & & & & \\
\hline \multicolumn{7}{c}{$\hat{\beta}_{i}^{c}$} & $\hat{\beta}_{i}^{d}$ & $\hat{\beta}_{i}^{d+}$ & $\hat{\beta}_{i}^{d-}$ & \\
\hline$\hat{\beta}_{i}^{c}$ & 1.0000 & & & \\
$\hat{\beta}_{i}^{d}$ & 0.3687 & 1.0000 & & & \\
$\hat{\beta}_{i}^{d+}$ & 0.1738 & 0.4193 & 1.0000 & & \\
$\hat{\beta}_{i}^{d-}$ & 0.1459 & 0.3889 & 0.0389 & 1.0000 & 1.0000 \\
$\bar{r}_{i}$ & -0.0114 & -0.0050 & 0.0104 & 0.0082 & \\
\hline \hline
\end{tabular}

‡ This table shows the descriptive statistics (Panel A) and time-series means of pairwise correlations (Panel B) for individual firm continuous betas, $\beta^{c}$, jump betas, $\beta^{d}$, positive and negative jump betas, $\beta^{d+}$ and $\beta^{d-}$, respectively, as well as the monthly excess returns, $\bar{r}$. All beta estimates are obtained using 5-minute data from previous 12-month using $\alpha_{i}=5 \sqrt{B V_{i}}$ thresholds, whereas $\bar{r}$ correspond to one-month-ahead excess returns. The estimates for all individual stocks and all calendar months are pooled together in calculating the statistics in this table.

Table 2: Average $R^{2}$ from jump regressions using 5 -min data across all stocks by year ${ }^{\ddagger}$

\begin{tabular}{ccccccc}
\hline \hline & \multicolumn{5}{c}{$\beta^{d+}$} & \multicolumn{3}{c}{$\beta^{d-}$} \\
\cline { 2 - 7 } Year/Threshold & 3 & 4 & 5 & 3 & 4 & 5 \\
\hline 2003 & 0.2213 & 0.3022 & 0.4633 & 0.1601 & 0.2142 & 0.2107 \\
2004 & 0.1079 & 0.1170 & 0.3343 & 0.1101 & 0.2084 & 0.4780 \\
2005 & 0.1208 & 0.1544 & 0.2677 & 0.1383 & 0.1387 & 0.2184 \\
2006 & 0.1647 & 0.3075 & 0.4240 & 0.1854 & 0.3252 & 0.5110 \\
2007 & 0.3673 & 0.5728 & 0.6721 & 0.3096 & 0.4501 & 0.5281 \\
2008 & 0.5242 & 0.6209 & 0.5674 & 0.5223 & 0.6732 & 1.0000 \\
2009 & 0.4243 & 0.5396 & 0.4888 & 0.3301 & 0.4581 & 0.6411 \\
2010 & 0.4834 & 0.6052 & 0.5597 & 0.5691 & 0.7425 & 0.8634 \\
2011 & 0.5044 & 0.5454 & 0.6051 & 0.6217 & 0.6730 & 0.8493 \\
\hline \hline
\end{tabular}

‡ This table shows the average $R^{2}$ from the jump regressions using the two asymmetric betas, $\beta^{d+}$ and $\beta^{d-}$, with three different thresholds across the all stocks in the data set. 
all the years in our sample; for the positive beta, $\beta^{d+}$, results are not always consistent. At the highest threshold level, $\alpha=5 \sqrt{B V}$, despite the low number of identified market jumps, the average $R^{2}$ from the jump regression approaches $80-90 \%$ after the GFC. This implies that albeit the low number of jumps, these few observations are extremely informative in explaining the variations in individual stock returns. This confirms an important stylised fact that the more extreme the market movements are (at higher threshold levels), the more congruent the individual stock returns are with the market (based on $R^{2}$ ). This particularly holds at the tails of return distribution. Further, we note that over the turmoil periods 2008-2011 corresponding to the US subprime and European sovereign debt crises, the interquantile ranges of $R^{2}$ s are substantially smaller for negative jumps than for positive jumps. This points to jump risk concentration among individual securities under extreme negative market conditions, while during positive market jumps the reaction of individual stocks is more heterogeneous, which in a portfolio setting will require less holdings to diversify such risk. ${ }^{17}$

The signature plots shown in Figures 2-3 justify our choice of 5-minute sampling frequency. The two figures provide exemplars that contrast the choice of sampling frequency for different levels of jump detection threshold, the least and the most turbulent years (2005 vs 2008) for defensive non-financial and financial securities (KO vs JPM). ${ }^{18}$ The difference in sensitivity of financial stocks to negative versus positive market jumps is especially pronounced during the crises periods (panels (e) vs (f) in Figure 3). We plot the continuous beta and three jump beta estimates calculated using data sampled at 1-second to 1800-second (i.e. 30-minute) frequencies. Correlations between asset returns decreases to zero as the sampling interval decreases, this is commonly known as the Epps effect in the literature. Hence the beta estimates would have downward bias when the sampling frequency is too high. Most

\footnotetext{
${ }^{17}$ Results tabulating IQRs of $R^{s} \mathrm{~S}$ from individual stock regressions are omitted here for brevity.

${ }^{18}$ Similar signature plots of the top and bottom 25 stocks in our sample (sorted by market cap) are available upon request.
} 
of the beta estimates suffer from Epps effect at higher frequencies, stabilize when the frequency is reduced down to 3-10 minutes, and destabilise for lower frequencies, confirming the optimal 5-minute choice.

In this section, we show that individual stock betas can vary greatly. Experienced investors will always consider allocating wealth in a number of assets rather than one single security. As the number of holdings increases, the range of portfolios betas will become more limited compared to the range of betas for individual securities, and will eventually converge to unity for an equally-weighted market benchmark. Given that investing in all securities listed on a broad market index may not be informationally efficient or cost effective, finding the optimal number of holdings in a portfolio to mitigate most of the jump risk is of significant importance. We discuss this in the next section.

\section{Portfolio Simulation}

The concept of portfolio diversification is straightforward: the level of portfolio risk falls as the number of holdings in a portfolio increases. ${ }^{19}$ In the previous section we explored the behaviour of continuous and jump risks for individual stocks. In this section we investigate how fast these systematic risks dissipate in portfolios.

In the last two decades, the availability of high-frequency data allowed new insights into portfolio diversification. For example, Silvapulle and Granger (2001) investigate asset correlations at the tails of return distributions and discuss the implications for portfolios of stocks. Bollerslev et al. (2013) examine the relationship between jumps in individual stocks and jumps in a market index and find that jumps occur more than three times as often at the individual stock level compared to an aggregate equally-weighted portfolio. This points to the fact that jumps may be diversifiable. In fact, using daily data, Pukthuanthong and

\footnotetext{
${ }^{19}$ Artzner et al. $(1997,1999)$ shows that this holds for any coherent risk measure.
} 
Figure 2: Signature plots of estimated betas for the Coca-Cola Co (KO) at different sampling frequencies and different thresholds used to identify jumps with $\alpha_{i}=3 \sqrt{B V_{i}}$ (top panel), $\alpha_{i}=4 \sqrt{B V_{i}}$ (middle panel) and $\alpha_{i}=5 \sqrt{B V_{i}}$ (bottom panel). For contrast, we present the results for 2005 (left column) and 2008 (right column). Sampling frequency on the $x$-axis is plotted on a logarithmic scale as in Hansen and Lunde (2006, pp.136,138, or 140).

(a) $2005, \alpha_{i}=3 \sqrt{B V_{i}}$

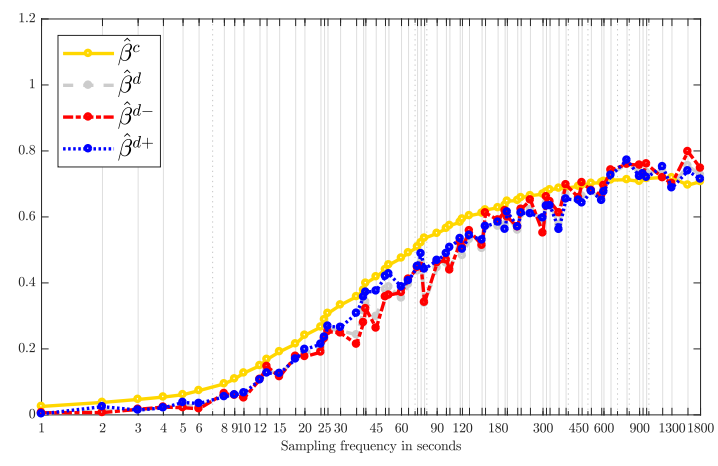

(c) $2005, \alpha_{i}=4 \sqrt{B V_{i}}$

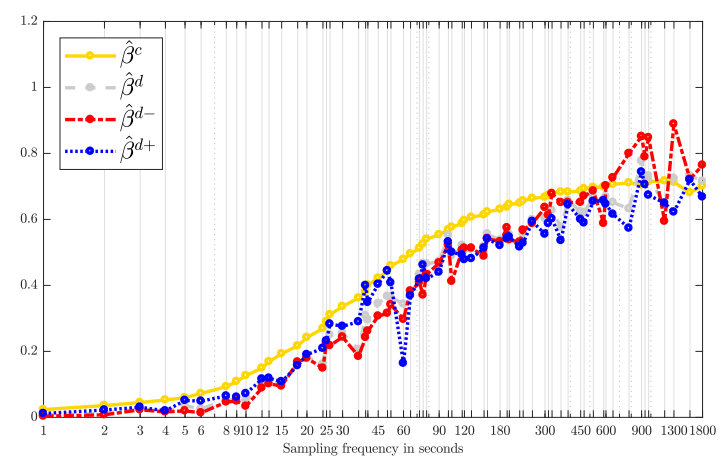

(e) 2005, $\alpha_{i}=5 \sqrt{B V_{i}}$

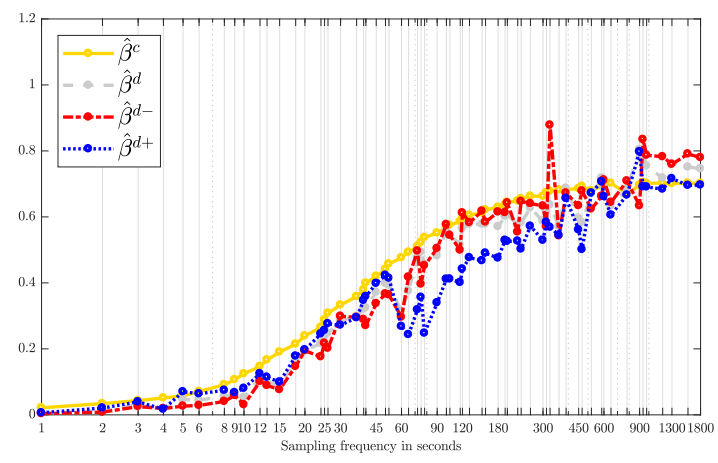

(b) $2008, \alpha_{i}=3 \sqrt{B V_{i}}$

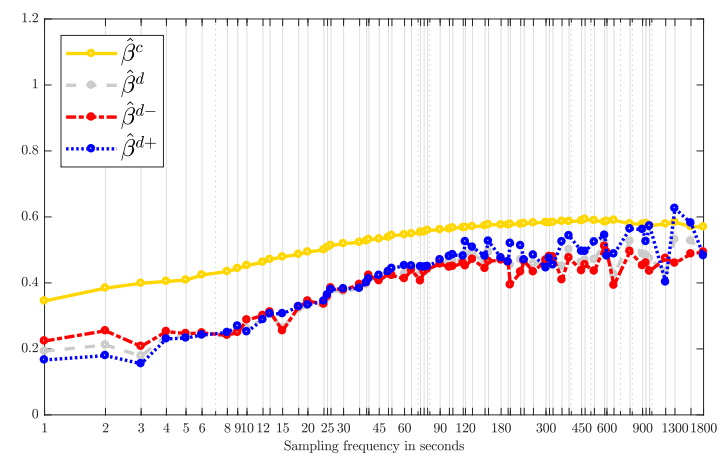

(d) 2008, $\alpha_{i}=4 \sqrt{B V_{i}}$

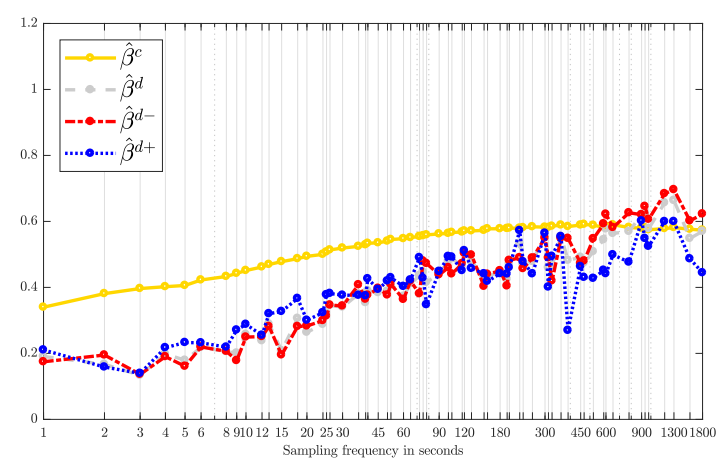

(f) $2008, \alpha_{i}=5 \sqrt{B V_{i}}$

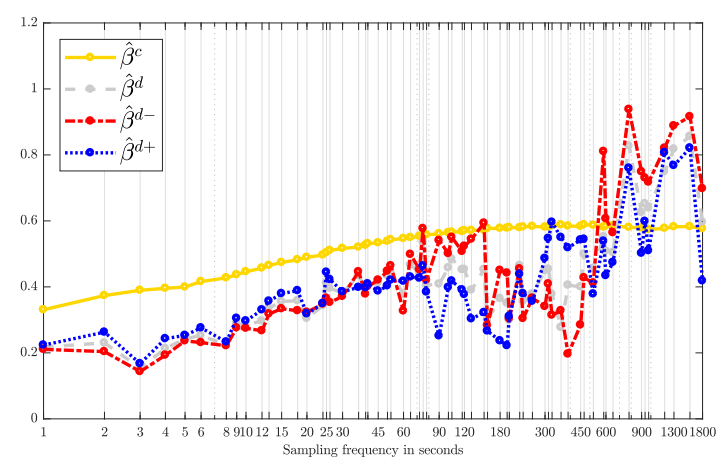


Figure 3: Signature plots of estimated betas for JPMorgan Chase \& Co (JPM) at different sampling frequencies and different thresholds used to identify jumps with $\alpha_{i}=3 \sqrt{B V_{i}}$ (top panel), $\alpha_{i}=4 \sqrt{B V_{i}}$ (middle panel) and $\alpha_{i}=5 \sqrt{B V_{i}}$ (bottom panel). For contrast, we present the results for 2005 (left column) and 2008 (right column). Sampling frequency on the $x$-axis is plotted on a logarithmic scale as in Hansen and Lunde (2006, pp.136,138, or 140). Note the difference in $y$-axis scales between panels for 2005 and 2008.

(a) $2005, \alpha_{i}=3 \sqrt{B V_{i}}$

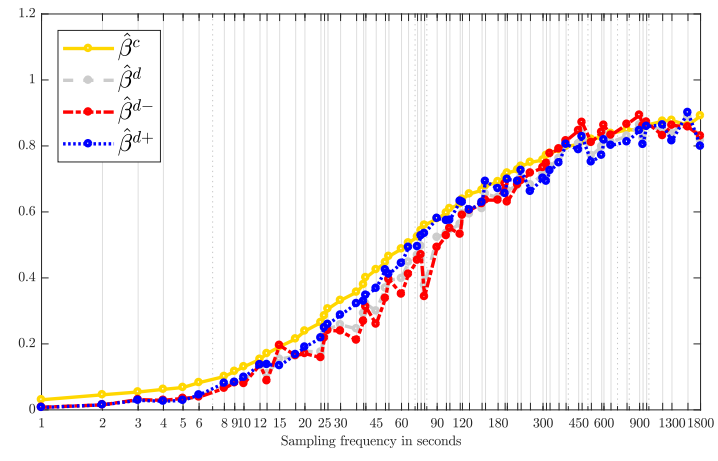

(c) $2005, \alpha_{i}=4 \sqrt{B V_{i}}$

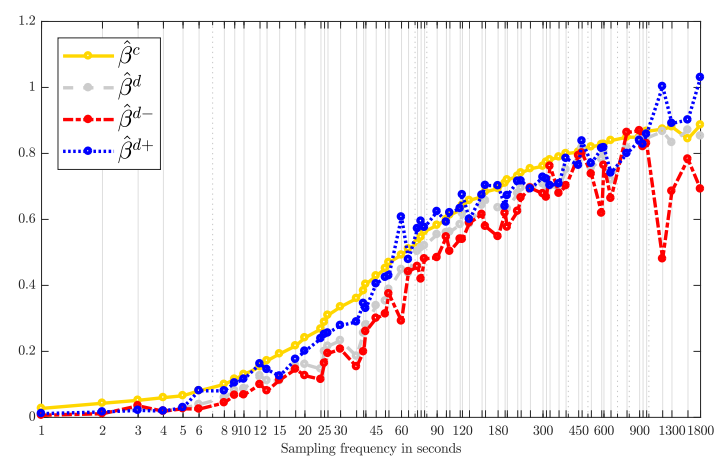

(e) $2005, \alpha_{i}=5 \sqrt{B V_{i}}$

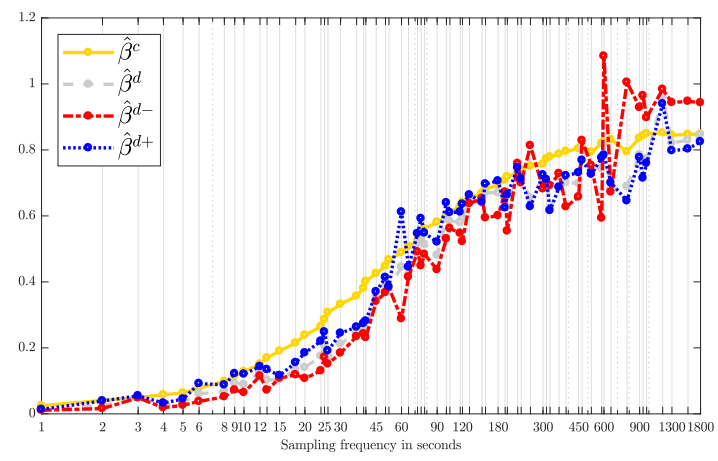

(b) $2008, \alpha_{i}=3 \sqrt{B V_{i}}$

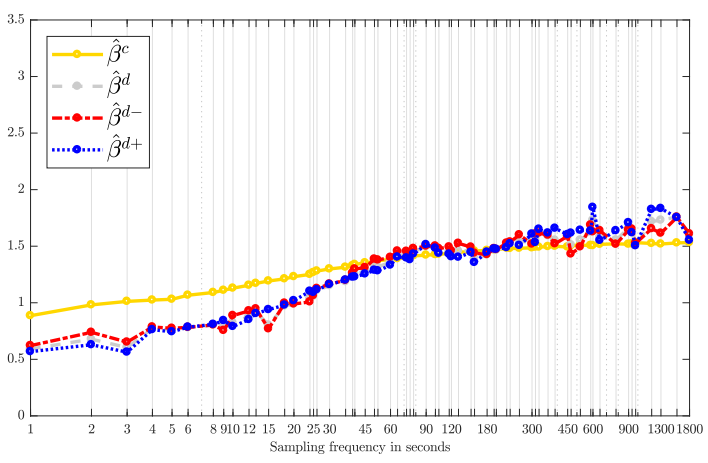

(d) 2008, $\alpha_{i}=4 \sqrt{B V_{i}}$

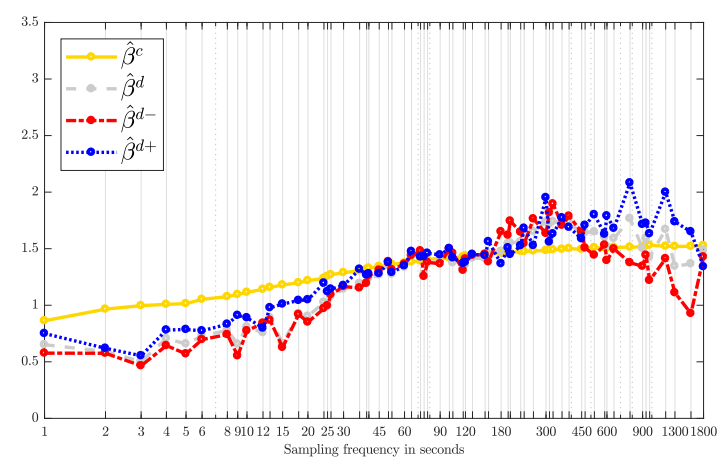

(f) $2008, \alpha_{i}=5 \sqrt{B V_{i}}$

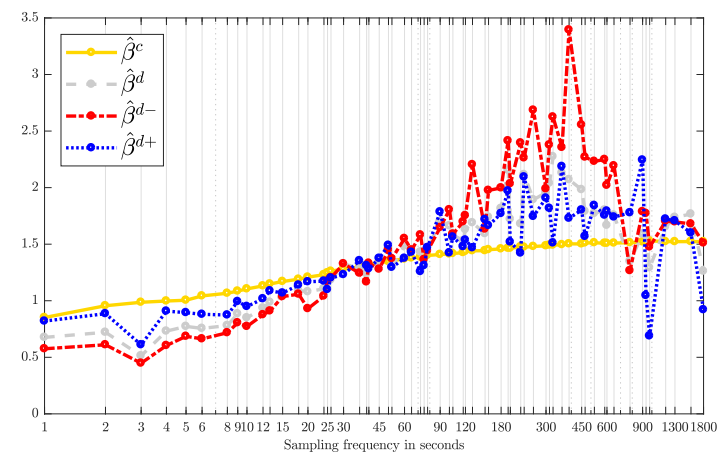


Roll (2014) consider implications of jumps for international diversification. Studies directly investigating optimal portfolio size using high-frequency data have only recently started to emerge (e.g., Alexeev and Dungey, 2015; Alexeev et al., 2016).

The use of higher-frequency data allows us to use methods suitable for direct estimation of sensitivity to extreme market changes via diffusion model with jumps and estimate systematic jump betas on a finer scale and without reliance on excessive estimation window lengths (i.e., 60 months in van Oordt and Zhou 2016). As in van Oordt and Zhou (2016), we find no consistent evidence of jump risk premia ${ }^{20}$, but note that the dispersion of portfolio jump betas is reduced with the number of portfolio holdings. The question of how many holdings could substantially reduce the variability of portfolio jump betas is our primary focus. We find that more holdings are required to stabilise portfolio sensitivity to extreme negative market shifts than the positive ones, and that this asymmetry is pronounced only during crises periods.

Using large-scale portfolio simulation, we contrast the variability in portfolio jump betas as the number of holdings in these portfolios changes. We analyse the spread of estimated betas in equi-weighted randomly constructed portfolios of different sizes, focusing on the difference in convergence between negative and positive jump betas as the number of holdings in portfolios increases. For investors, the knowledge that individual stocks respond differently to the positive and negative extreme events is likely to be valuable in developing portfolio risk management strategies. However, investors, typically holding several stocks, may be rightfully concerned with the overall exposure of their portfolios to systematic jump risk. Moreover, investors exhibit different attitudes towards extreme gains and extreme losses. We assert that if an asset tends to move downwards in a declining market

\footnotetext{
${ }^{20}$ We used the two-stage regression by Fama and MacBeth (1973) to estimate the risk premia for both the symmetric and asymmetric jump betas. Results are omitted here for brevity but available upon request.
} 
more than it moves upward in a rising market, such asset is unattractive to hold, especially during market downturns when wealth of investors is low.

Using a 12-month estimation window, for each year from 2003 to 2011 we construct 5,000 random equally-weighted portfolios with the number of holdings in each portfolio ranging from 1 to 200. For each of these portfolios we estimate several systematic discontinuous risks - symmetric jump beta, $\beta^{d}$, as well as $\beta^{d+}$ and $\beta^{d-} .{ }^{21}$ We assess the stability of the systematic portfolio risks by analyzing the inter-quartile ranges of the beta distributions as the number of stocks in portfolio increases. Defined as the difference between two percentiles, $75 \%$ and $25 \%$, the inter-quartile range (IQR) is a stable measure that is robust to outliers. That is,

$$
I Q R_{(n)} \equiv E D F_{(n)}^{-1}(.75)-E D F_{(n)}^{-1}(.25),
$$

where $E D F_{(n)}$ is the empirical distribution function of the estimated betas $\left(\beta^{d}, \beta^{d+}\right.$ or $\left.\beta^{d-}\right)$ for randomly drawn $n$-stock portfolios.

Figure 4 depicts the typical distributions of $\beta^{d-}$ and $\beta^{d+}$ for equally weighted randomly drawn portfolios of $n=1, \ldots, 200$ stocks for the year of $2008 .{ }^{22}$ Since these central ranges are dependent on the particular time period, for each year in our analysis, we normalize the IQR for the $n$-stock portfolios and represent it as a fraction of the IQR of the singlestock portfolio. This approach was first introduced in Alexeev et al. (2016). The normalized IQRs, or $I Q R_{(n)} / I Q R_{(1)}$, of $\hat{\beta}^{d}, \hat{\beta}^{d+}$ and $\hat{\beta}^{d-}$ for year 2008 are depicted in Figure 5 for $n=$ $1, \ldots, 200$. Since the market index is an equally-weighted portfolio consisting of all investible stocks, and is thus unique, it has $I Q R_{(N)}=0$. As a result, the normalized IQRs in Figure 5 are bounded between 0 and 1 . We find that the difference among the normalized IQRs for

\footnotetext{
${ }^{21}$ This amounts to 9 years $\times(199 \times 5,000+500)=8,959,500$ single-year portfolio return vectors.

${ }^{22}$ Results for other years exhibit similar patterns and are omitted for brevity. These results are available upon request.
} 
Figure 4: Distribution of $\beta^{d-}$ (left panel) and $\beta^{d+}$ (right panel) across portfolio sizes. Red points represent maxima and minima, black lines represent interpercentaile range from $2.5 \%$ to $97.5 \%$, blue lines denote interguantile range and the black circles are the medians of the distributions. We use 2008 data in estimating the results in this figure.
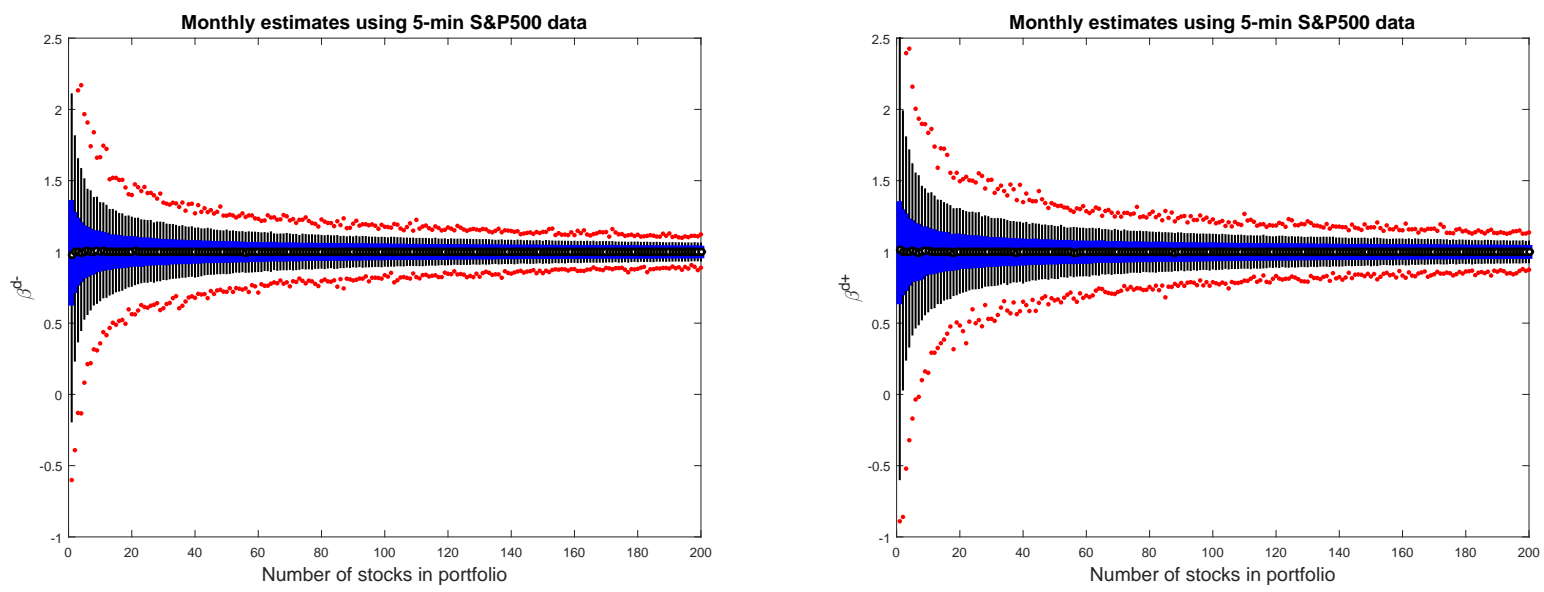

the three different betas are more pronounced during periods of high volatility and for more extreme events (consider the top versus the bottom panel in Figure 5). Figure 5 shows that the IQR of portfolio jump betas decrease substantially as the number of stocks in the portfolio, $n$, increases. The use of the normalized IQRs enables us to contrast the required portfolio sizes at different periods of time, in order to achieve the same proportional reduction in IQRs of beta for these portfolios relative to the beta spreads of individual securities. In discussing our findings, we consider a fivefold reduction in the spread of portfolio jump risk component relative to the jump beta spread of individual securities (represented by the horizontal line at 0.2 in Figure 5).

Table 3 outlines the required portfolio sizes in order to reduce the normalized IQR five fold. We examine each year separately and consider several threshold levels for jump identification. It is evident that during periods of market distress characterised by high volatility, the number of stocks required to reduce the IQR and stabilise the negative jump beta is considerably higher than during the less volatile periods. During periods of normal market 
Figure 5: Normalized IQR of betas across portfolio sizes. Both panels display results based on year 2008. The top panel displays results based on a threshold $\alpha_{i}=3 \sqrt{B V_{i}}$ and the bottom panel is based on $\alpha_{i}=5 \sqrt{B V_{i}}$. As can be observed from the figures below, the asymmetry in signed betas is more pronounced when more extreme events are considered. The optimal number of holdings in a portfolio is determined at the intersection of the normalised IQR curve (red, blue and black) with the desired level of variability reduction (in this case 0.2 denoted by horizonal purple line).
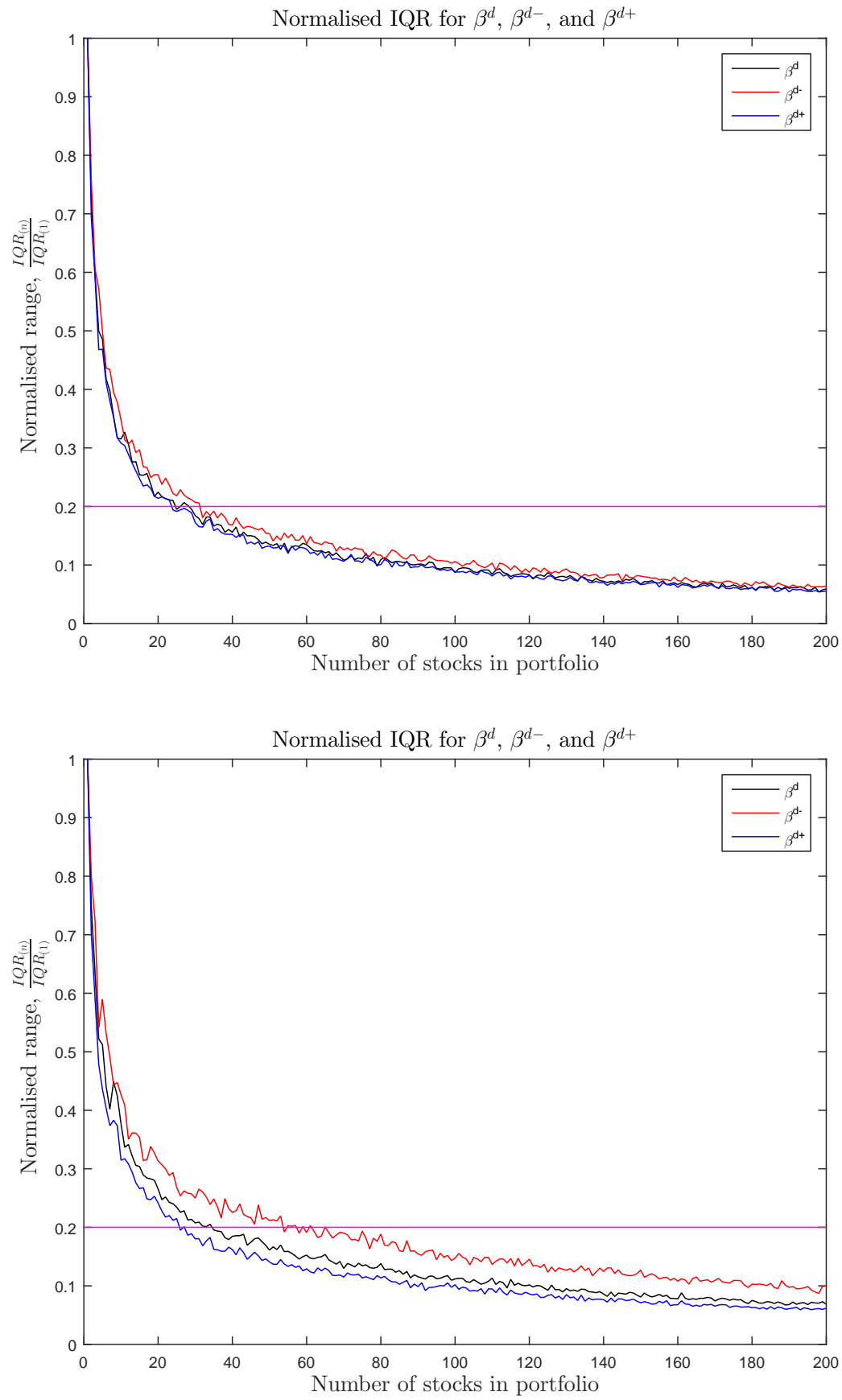
activity, there is little distinction between negative and positive jump risk and, consequently, the recommended portfolio sizes. The difference in the number of stocks required in order to achieve the same proportionate reduction increases substantially in 2008 and 2011. When we consider only negative market jumps, our recommended portfolio sizes are greater than when both negative and positive jumps are considered (the symmetric jump risk). This discrepancy is more pronounced during periods of increased market volatility. This asymmetry effect is most vividly observed when we consider only the most extreme events (last three columns of Table 3). For example, when the threshold is $\alpha_{i}=5 \sqrt{B V_{i}}$, during normal market times this discrepancy ranges from 3 to 7 stocks, while in 2008 and 2011 there are 17 to 19 stocks' difference. Ignoring the asymmetry in sensitivities to negative versus positive market jumps lead to under-diversification of portfolios, and hence increased exposure to extreme negative market shifts. Without considering asymmetric betas, the optimal portfolio sizes are 35 and 34 in 2008 and 2011 for threshold $\alpha_{i}=5 \sqrt{B V_{i}}$, respectively. However, if investor is concerned with extreme negative shifts in the market, it would be advisable to hold 54 and 51 stocks instead, to reduce the sensitivity of portfolio returns to extreme negative market shifts compared to a single stock portfolio.

\section{Conclusion}

In this paper, we studied jump dependence between processes using high-frequency data focusing on observations that are informative for jump inference. In particular, we investigated the relationship between jumps in a process for an asset (or portfolio of assets) and an aggregate market factor, and analysed co-movements in jumps of the two processes. We examined a linear relationship between jumps and assessed the sensitivity of jumps in (portfolios of) assets to jumps in the market. Using high-frequency data for the constituents of the S\&P500 index over the period 2 January 2003 to 30 December 2011, we estimated jump 
Table 3: Portfolio sizes, $n$, required to reduce normalised IQR, or $I Q R_{(n)} / I Q R_{(1)}$, to 0.2.

\begin{tabular}{cccccccccc}
\hline \hline Year & \multicolumn{3}{c}{$\alpha_{i}=3 \sqrt{B V_{i}}$} & \multicolumn{3}{c}{$\alpha_{i}=4 \sqrt{B V_{i}}$} & \multicolumn{3}{c}{$\alpha_{i}=5 \sqrt{B V_{i}}$} \\
& $\beta^{d}$ & $\beta^{d-}$ & $\beta^{d+}$ & $\beta^{d}$ & $\beta^{d-}$ & $\beta^{d+}$ & $\beta^{d}$ & $\beta^{d-}$ & $\beta^{d+}$ \\
\hline 2003 & 30 & 31 & 30 & 36 & 38 & 35 & 36 & 39 & 35 \\
2004 & 31 & 33 & 31 & 36 & 37 & 36 & 34 & 40 & 33 \\
2005 & 30 & 31 & 29 & 35 & 36 & 36 & 35 & 40 & 33 \\
2006 & 30 & 30 & 29 & 34 & 35 & 34 & 33 & 39 & 32 \\
2007 & 28 & 29 & 28 & 32 & 33 & 32 & 32 & 39 & 30 \\
2008 & 25 & 32 & 24 & 29 & 36 & 25 & 35 & 54 & 26 \\
2009 & 30 & 31 & 28 & 34 & 36 & 33 & 34 & 41 & 31 \\
2010 & 31 & 31 & 31 & 35 & 37 & 34 & 35 & 42 & 32 \\
2011 & 24 & 30 & 23 & 29 & 40 & 28 & 34 & 51 & 27 \\
\hline
\end{tabular}

betas for the negative and positive market shifts separately, and considered implications for portfolio risk management using upside and downside jump betas as risk factors.

We find that the number of stocks required to stabilize portfolio exposure to sudden large negative price changes in the market is substantially greater than under positive one or if the asymmetry is not taken into account. Our empirical findings suggest that holding 54 stocks is advisable to substantially reduce the IQR of the portfolio sensitivity to extreme market downturn. This number is more than twice the number of stocks (26 stocks) required to induce similar reduction in portfolio sensitivity to positive market shifts. Moreover, if the asymmetry is ignored, the recommended portfolio size is 35 stocks leaving portfolios exposed to extreme negative events. These conservative recommendations are based on the 2008 results - uncountably, the worst case scenario. Indeed, the asymmetry is more pronounced for the most extreme events - with high jump identification thresholds - occurring during periods of very high market volatility, in our case 2008 and 2011. 
Our results provide important guidelines on the identification of an optimal size of equity portfolio to stabilize portfolio jump betas. These findings have important implications for fund managers. For instance, for leveraged and inverse leveraged ETFs, which are designed to generate multiple times (either positive or negative) the return of indices they are tracking, generating the promised returns relies on minimising the tracking errors. In fact, evidence suggests that historical tail betas are persistent and capture future systematic risk. This feature points to the importance of tail betas as factors in portfolio management process.

The findings in this paper suggest some interesting future developments. First, it would be interesting to investigate the behaviour of jump betas during "flash crashes", short unpredictable periods between an extreme "low" and extreme "high". Second, our findings of tighter fits in individual stock regressions during negative market jumps points towards important asymmetries when exploring co-jumping behaviour. This will highlight significant common jumps between correlated stochastic processes, and allow to distinguish among contemporaneous, permanent and exogenous co-jump events. This is part of the ongoing research agenda.

\section{References}

Aït-Sahalia, Yacine and Jean Jacod, "Analyzing the Spectrum of Asset Returns: Jump and Volatility Components in High Frequency Data," Journal of Economic Literature, 2012, 50 (4), 1007-50.

_, Per A. Mykland, and Lan Zhang, "Ultra High Frequency Volatility Estimation with Dependent Microstructure Noise," Journal of Econometrics, 2011, 160 (1), 160 - 175.

Alexeev, Vitali and Mardi Dungey, "Equity Portfolio Diversification with High Frequency Data," Quantitative Finance, 2015, 15 (7), 1205-1215.

- , - , and Wenying Yao, "Continuous and Jump Betas: Implications for Portfolio Diversification," Econometrics, 2016, 4 (2), 27. 
$\ldots, \ldots$, and $\_,$"Time-varying continuous and jump betas: The role of firm characteristics and periods of stress," Journal of Empirical Finance, 2017, 40, 1 - 19.

Andersen, Torben G., Dobrislav Dobrev, and Ernst Schaumburg, "Jump-robust volatility estimation using nearest neighbor truncation," Journal of Econometrics, 2012, 169 (1), 75 93. Recent Advances in Panel Data, Nonlinear and Nonparametric Models: A Festschrift in Honor of Peter C.B. Phillips.

_ , Tim Bollerslev, and Francis X. Diebold, “Roughing It Up: Including Jump Components in the Measurement, Modeling, and Forecasting of Return Volatility," Review of Economics and Statistics, 2007, (4), 701-720.

_ , _ , _, and Ginger Wu, Realized Beta: Persistence and Predictability, Vol. 20 of Econometric Analysis of Financial and Economic Time Series (Advances in Econometrics), Emerald Group Publishing Ltd,

Ang, Andrew, Joseph Chen, and Yuhang Xing, "Downside Risk," Review of Financial Studies, 2006, 19 (4), 1191-1239.

Artzner, Philippe, Freddy Delbaen, Jean-Marc Eber, and David Heath, "Thinking Coherently," Risk, 1997, 10, 68-71.

_ , , , , and _ , "Coherent Measures of Risk," Mathematical Finance, 1999, 9 (3), 203-228.

Audrino, Francesco and Yujia Hu, "Volatility Forecasting: Downside Risk, Jumps and Leverage Effect," Econometrics, 2016, 4 (1), 8.

Bajgrowicz, Pierre, Olivier Scaillet, and Adrien Treccani, "Jumps in High-Frequency Data: Spurious Detections, Dynamics, and News," Management Science, 2016, 62 (8), 2198-2217.

Bandi, R.M and J.R. Russell, "Separating Microstructure Noise from Volatility," Journal of Financial Economics, 2006, 79, 655-692.

Barberis, Nicholas, "The Psychology of Tail Events: Progress and Challenges," American Economic Review, 2013, 103 (3), 611-616.

Barndorff-Nielsen, Ole E. and Neil Shephard, "Power and Bipower Variation with Stochastic Volatility and Jumps," Journal of Financial Econometrics, 2004, 2 (1), 1-37.

_ and _ , "Econometrics of Testing for Jumps in Financial Economics Using Bipower Variation," Journal of Financial Econometrics, 2006, 4 (1), 1-30.

_ , Silja Kinnebrock, and Neil Shephard, "Measuring Downside Risk Ü Realized Semivariance," in Tim Bollerslev, Jeffrey Russell, and Mark Watson, eds., Volatility and Time Series Econometrics: Essays in Honor of Robert Engle, Oxford University Press, 2010.

Barro, Robert J., "Rare Disasters and Asset Markets in the Twentieth Century," The Quarterly Journal of Economics, 2006, 121 (3), 823-866. 
Bates, David S., "The Market for Crash Risk," Journal of Economic Dynamics and Control, 2008, 32 (7), 2291-2321.

Black, Angela, Jing Chen, Oleg Gustap, and Julian M Williams, "The Importance of Jumps in Modelling Volatility during the 2008 Financial Crisis," Technical Report July 2012.

Bollerslev, Tim and Viktor Todorov, "Tails, Fears, and Risk Premia," The Journal of Finance, 2011, 66 (6), 2165-2211.

_, Sophia Zhengzi Li, and Viktor Todorov, "Roughing up beta: Continuous versus discontinuous betas and the cross section of expected stock returns," Journal of Financial Economics, 2016, 120 (3), 464-490.

- , Tzuo Hann Law, and George Tauchen, "Risk, Jumps, and Diversification," Journal of Econometrics, 2008, 144 (1), 234-256.

_ , Viktor Todorov, and Sophia Zhengzi Li, "Jump Tails, Extreme Dependencies, and the Distribution of Stock Returns," Journal of Econometrics, 2013, 172 (2), 307 - 324. Latest Developments on Heavy-Tailed Distributions.

Christensen, Kim, Roel C.A. Oomen, and Mark Podolskij, "Fact or Friction: Jumps at Ultra High Frequency," Journal of Financial Economics, 2014, 114 (3), 576-599.

Davies, Robert and George Tauchen, "Data-driven Jump Detection Thresholds for Application in Jump Regressions," ERID Working Paper Number 213, Duke University September 2015.

Dungey, Mardi, Matteo Luciani, and David Veredas, "Ranking Systemically Important Financial Institutions," Working Papers ECARES 2013/130530, ULB - Universite Libre de Bruxelles 2012.

Embrechts, Paul, Claudia Kluppelberg, and Thomas Mikosch, Modelling Extremal Events for Insurance and Finance, Springer, 1997.

Epps, Thomas W., "Comovements in Stock Prices in the Very Short Run," Journal of the American Statistical Association, 1979, 74 (366), 291-298.

Fama, Eugene F. and James D. MacBeth, "Risk, Return, and Equilibrium: Empirical Tests," Journal of Political Economy, 1973, 81 (3), 607-636.

Fisher, Lawrence, "Some New Stock-Market Indexes," The Journal of Business, 1966, 39 (1), 191-225.

Forbes, Kristin J. and Roberto Rigobon, "No Contagion, Only Interdependence: Measuring Stock Market Comovements," The Journal of Finance, 2002, 57 (5), 2223-2261.

Fry, Renee, Vance L. Martin, and Chrismin Tang, "A New Class of Tests of Contagion With Applications," Journal of Business \& Economic Statistics, 2010, 28 (3), 423-437. 
Giorgi, Enrico G. De and Shane Legg, "Dynamic Portfolio Choice and Asset Pricing with Narrow Framing and Probability Weighting," Journal of Economic Dynamics and Control, 2012, 36 (7), 951-972.

Guo, Hui, Kent Wang, and Hao Zhou, "Good Jumps, Bad Jumps, and Conditional Equity Premium," Working Paper, February 2015.

Hansen, Peter R and Asger Lunde, "Realized Variance and Market Microstructure Noise," Journal of Business \& Economic Statistics, 2006, 24 (2), 127-161.

Jacod, Jean, "Asymptotic Properties of Realized Power Variations and Related Functionals of Semimartingales," Stochastic Processes and their Applications, 2008, 118 (4), 517 - 559.

- and Philip Protter, Discretization of Processes, Springer Berlin Heidelberg, 2012.

- and Viktor Todorov, "Testing for Common Arrivals of Jumps for Discretely Observed Multidimensional Processes," Ann. Statist., 2009, 37 (4), 1792-1838.

Lahaye, Jerome, Sebastien Laurent, and Christopher J. Neely, "Jumps, Cojumps and Macro Announcements," Journal of Applied Econometrics, 2011, 26 (6), 893-921.

Li, Jia, Viktor Todorov, and George Tauchen, "Jump regressions," Econometrica, 2017, 85 (1), 173-195.

_ , _ , , and Rui Chen, "Mixed-scale Jump Regressions with Bootstrap Inference," Working Paper, Duke University March 2015.

Liu, Lily Y., Andrew J. Patton, and Kevin Sheppard, "Does anything beat 5-minute RV? A comparison of realized measures across multiple asset classes," Journal of Econometrics, jul 2015, 187 (1), 293-311.

Mancini, Cecilia, "Disentangling the Jumps of the Diffusion in a Geometric Jumping Brownian Motion," Giornale dell'Istituto Italiano degi Attuari, 2001, 64, 19-47.

_ , "Non-parametric Threshold Estimation for Models with Stochastic Diffusion Coefficient and Jumps," Scandinavian Journal of Statistics, 2009, 36 (2), 270-296.

- and Fabio Gobbi, "Identifying the Brownian Covariation from the Co-jumps Given Discrete Observations," Econometric Theory, 4 2012, 28 (2), 249-273.

- and Roberto Renò, "Threshold Eestimation of Markov Models with Jumps and Interest Rate Modeling," Journal of Econometrics, jan 2011, 160 (1), 77-92.

Markowitz, H.M., Portfolio Selection: Efficient Diversification of Investments Cowles Commission for Research in Economics: Monographs, Yale University Press, 1971.

Mykland, Per A. and Lan Zhang, "Between Data Cleaning and Inference: Pre-averaging and Robust Estimators of the Efficient Price," Journal of Econometrics, 2016, 194 (2), 242-262. 
Patton, Andrew J. and Michela Verardo, "Does Beta Move with News? Firm-specific Information Flows and Learning About Profitability," Review of Financial Studies, 2012, 25, 2789-2839.

Pukthuanthong, K. and R. Roll, "Internationally Correlated Jumps," Review of Asset Pricing Studies, 2014, 5 (1), 92-111.

Rietz, Thomas A., "The Equity Risk Premium: A Solution," Journal of Monetary Economics, 1988, 22 (1), $117-131$.

Rigobon, Roberto, "Contagion, Spillover and Interdependence," Bank of England Working Paper, 2016.

Silvapulle, P. and C.W.J. Granger, "Large Returns, Conditional Correlation and Portfolio Diversification: A Value-at-Risk Approach," Quantitative Finance, 2001, 1 (5), 542-551.

Todorov, Viktor and Tim Bollerslev, "Jumps and Betas: A New Framework for Disentangling and Estimating Systematic Risks," Journal of Econometrics, 2010, 157 (2), 220-235.

van Oordt, Maarten R. C. and Chen Zhou, "Systematic Tail Risk," Journal of Financial and Quantitative Analysis, 2016, 51 (02), 685-705.

Visser, M. P., "Forecasting S\&P 500 daily volatility using a proxy for downward price pressure," Working paper, University of Amsterdam 2008.

Wasserfallen, Walter and Heinz Zimmermann, "The behavior of intra-daily exchange rates," Journal of Banking \& Finance, 1985, 9 (1), 55-72. 